\title{
JORDAN PROPERTIES OF AUTOMORPHISM GROUPS OF CERTAIN OPEN ALGEBRAIC VARIETIES
}

\author{
TATIANA BANDMAN AND YURI G. ZARHIN
}

\begin{abstract}
Let $W$ be a quasiprojective variety over an algebraically closed field of characteristic zero. Assume that $W$ is birational to a product of a smooth projective variety $A$ and the projective line. We prove that if $A$ contains no rational curves then the automorphism group $G:=\operatorname{Aut}(W)$ of $W$ is Jordan. That means that there is a positive integer $J=J(W)$ such that every finite subgroup $\mathcal{B}$ of $G$ contains a commutative subgroup $\mathcal{A}$ such that $\mathcal{A}$ is normal in $\mathcal{B}$ and the index $[\mathcal{B}: \mathcal{A}] \leq J$.
\end{abstract}

\section{INTRODUCTION}

Throughout this paper $k$ is an algebraically closed field of characteristic zero. All varieties, if not indicated otherwise, are irreducible, algebraic, and defined over $k$. If $X$ is an algebraic variety over $k$ then we write $\operatorname{Aut}(X)$ for its group of (biregular) automorphisms and $\operatorname{Bir}(X)$ for its group of birational automorphisms. As usual, $\mathbb{P}^{n}$ stands for the $n$-dimensional projective space and $\mathbb{A}^{n}\left(\mathbb{A}_{x_{1}, \ldots, x_{n}}^{n}\right)$ for the $n$-dimensional affine space (with coordinates $x_{1}, \ldots, x_{n}$, respectively).

The definition of a Jordan group was introduced in [Po1].

Definition 1.1. A group $\mathcal{G}$ is called Jordan [Po1] if there exists a positive integer $J$ that enjoys the following property. Every finite subgroup $\mathcal{B}$ of $\mathcal{G}$ contains a commutative subgroup $\mathcal{A}$ such that $\mathcal{A}$ is normal in $\mathcal{B}$ and the index $[\mathcal{B}: \mathcal{A}] \leq J$. Such a smallest $J$ is called the Jordan index of $\mathcal{G}$ and denoted by $J_{\mathcal{G}}$.

Definition 1.2. Let $G$ be a group.

(a) $G$ is called bounded [Po2, PS1] if there is a positive integer $C=C_{G}$ such that the order of every finite subgroup of $G$ does not exceed $C$.

(b) $G$ is called quasi-bounded if there is a nonnegative integer $a:=a(G)$ such that each finite abelian subgroup of $G$ is generated by at most $A$ elements.

(c) $G$ is called strongly Jordan [PS2, BZ2] if it is Jordan and quasi-bounded.

Remark 1.3. (i) If

$$
\{0\} \rightarrow G_{1} \rightarrow G \rightarrow G_{2} \rightarrow\{0\}
$$

2010 Mathematics Subject Classification. 14J50, 14E07, 14J27, 14L30, 14J30, 14 K05.

The second named author is partially supported by a grant from the Simons Foundation (\#246625 to Yuri Zarkhin). Part of this work was done in May-June 2016 during his stay at the Max-PlanckInstitut für Mathematik, whose hospitality and support are gratefully acknowledged. 
is a short exact sequence of groups and both $G_{1}$ and $G_{2}$ are bounded (resp. quasi-bounded) then one may easily check that $G$ is also bounded (resp. quasibounded). Indeed, let $H$ be a finite (resp. finite abelian) subgroup of $G$. Let $H_{2}$ be the image of $H$ in $G_{2}$ and $H_{1}$ the intersection of $H$ and the kernel of $G \rightarrow G_{2}$. Then $H$ sits in the short exact sequence

$$
\{0\} \rightarrow H_{1} \rightarrow H \rightarrow H_{2} \rightarrow\{0\}
$$

where $H_{i}$ is a finite (resp. finite abelian) subgroup of $G_{i}, i=1,2$. If both $G_{1}$ and $G_{2}$ are bounded then the order of $H$ does not exceed $C_{G_{1}} C_{G_{2}}$, i.e., $G$ is also bounded. If both $G_{1}$ and $G_{2}$ are quasi-bounded then $H$ is generated by, at most, $a\left(G_{1}\right)+a\left(G_{2}\right)$ elements [MZ, Lemma 2.3].

(ii) If both $G_{1}$ and $G_{2}$ are Jordan then $G$ does not have to be Jordan.

(iii) Clearly, every subgroup of a (strongly) Jordan group is also (strongly) Jordan.

The group $\mathrm{GL}_{n}(\mathbb{Z})$ is bounded by Minkowski's Theorem ([Ser, Sect. 9.1]). The classical theorem of Jordan ([CR, Sect. 36], [Ser, Sect. 9.2], [MuTu]) asserts that $\operatorname{GL}(n, k)$ is strongly Jordan. An example of a non Jordan group is given by $\operatorname{GL}\left(n, \overline{\mathbb{F}}_{p}\right)$ where $\overline{\mathbb{F}}_{p}$ is the algebraic closure of a finite field $\mathbb{F}_{p}$ and $n \geq 2$.

We refer the reader to [Po2] for references and survey on this topic.

Let $X$ be an algebraic variety over $k$. It is known that $\operatorname{Aut}(X)$ is Jordan if either $\operatorname{dim}(X) \leq 2[$ Po1, BZ1] or $X$ is projective [MZ]. It is also known ([PS1] combined with $[\operatorname{Bir}])$, that if $X$ is an irreducible variety then $\operatorname{Bir}(X)$ is Jordan if either $q(X)=0$ or $X$ is not uniruled (in particular, Cremona groups $\operatorname{Bir}\left(\mathbb{P}^{N}\right)$ and $\operatorname{groups} \operatorname{Aut}\left(\mathbb{A}^{N}\right)$ are Jordan).

On the other hand, $\operatorname{Bir}(X)$ is not Jordan if $X$ is birational to a product $A \times \mathbb{P}^{n}$ where $n \geq 1$ and $A$ is a positive-dimensional abelian variety over $k$ [Za1].

Since $\operatorname{Aut}(X)$ is a subgroup of $\operatorname{Bir}(X)$, it is Jordan whenever $\operatorname{Bir}(X)$ is Jordan. But $\operatorname{Aut}(X)$ may be Jordan when $\operatorname{Bir}(X)$ is not. To the best of our knowledge, there is no example of an algebraic variety with non-Jordan automorphisms group. The aim of this paper is to prove the Jordan property of the group $\operatorname{Aut}(X)$ for open subsets of certain uniruled varieties.

Definition 1.4. We call a smooth projective variety $A$ rigid if it is irreducible and contains no rational curves.

We prove the following

Theorem 1.5. Let $W$ be an irreducible quasiprojective variety that is birational to a product $A \times \mathbb{P}^{1}$ where $A$ is a smooth rigid projective variety. Then $\operatorname{Aut}(W)$ is strongly Jordan.

The case of $\operatorname{dim}(W)=2, \operatorname{dim}(A)=1$ was done in [Po1, Za2, BZ1].

The case of $\operatorname{dim}(W)=3$ was studied in [Za1, PS0, PS1, BZ2, PS2]. Here is the final answer for $\operatorname{Bir}(X)$ [PS2. Let $X$ be a threefold. Then $\operatorname{Bir}(X)$ is not Jordan if 
and only if either $X$ is birational to $E \times \mathbb{P}^{2}$, where $E$ is an elliptic curve, or $X$ is birational to $S \times \mathbb{P}^{1}$, where $S$ is one of the following:

Case 1. An abelian surface;

Case 2. A bielliptic surface;

Case 3. A surface with Kodaira dimension $\varkappa(S)=1$ such that the Jacobian fibration of the pluricanonical fibration is locally trivial in Zariski topology.

Thus, Theorem 1.5 leads to the following

Corollary 1.6. Assume that $W$ is a quasiprojective irreducible variety of dimension $d \leq 3$. Assume that $W$ is not birational to $E \times \mathbb{P}^{2}$, where $E$ is an elliptic curve. Then Aut $(W)$ is Jordan.

Remark 1.7. Let $W$ be a (nonempty) irreducible algebraic variety over $k$ and $W^{\text {ns }} \subset$ $W$ the open dense (sub)set of its nonsingular points. Then $u\left(W^{\mathrm{ns}}\right) \subset W^{\mathrm{ns}}$ for each $u \in$ $\operatorname{Aut}(W)$. This gives rise to the natural group homomorphism $\operatorname{Aut}(W) \rightarrow \operatorname{Aut}\left(W^{\mathrm{ns}}\right)$, which is injective, since $W^{\text {ns }}$ is dense in $W$ in Zariski topology. This implies that in the course of the proof of Theorem [1.5 and Corollary [1.6 we may assume that $W$ is smooth.

The paper is organized as follows. Section 2 contains notation and auxiliary results about fiberwise automorphisms of fibered varieties. In Section 3 we discuss automorphism groups of varieties that are birational to a product $A \times \mathbb{P}^{1}$ where $A$ is a smooth rigid projective variety. Section 4 contains the proof of Theorem 1.5 and Corollary 1.6

1.1. Acknowledgements. We are grateful to Shulim Kaliman, Michel Brion, and Vladimir Berkovich for helpful discussions. Our thanks go to the referees, whose comments helped to improve the exposition.

\section{Preliminaries}

If $X$ is an irreducible algebraic variety over $k$ then

- We write $k[X]$ for the ring of regular functions on $X$ and $k(X)$ for its field of rational functions. In this case one may view $\operatorname{Bir}(X)$ as the group of all $k$-linear automorphisms of $k(X)$ and $\operatorname{Aut}(X)$ as a certain subgroup of $\operatorname{Bir}(X)$. We write $\operatorname{id}_{X}$ for the identity automorphism of $X$, which may be viewed as the identity element of groups $\operatorname{Aut}(X)$ and $\operatorname{Bir}(X)$.

- By points of $X$ (unless otherwise stated) we always mean $k$-points. A general point means a point of an open dense subset of $X$.

- If $X$ is smooth then $K_{X}$ and $q(X)$ stand for the canonical class of $X$ and irregularity $h^{0,1}(X)$ of $X$, respectively.

- $\mathbb{C}, \mathbb{Q}$ and $\mathbb{Z}$ stand for fields of complex numbers, the rationals, and ring of integers, respectively.

- If $F$ is a field then we write $\bar{F}$ for its algebraic closure. 
- Let $X, Y, T$ be irreducible varieties, $p: X \rightarrow T, q: Y \rightarrow T$ morphisms. We say that a rational map $f: X \rightarrow Y$ is $p, q$-fiberwise if there exists morphism $g_{f}: T \rightarrow T$ such that the following diagram commutes:

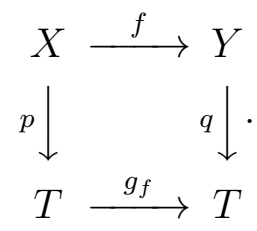

- If $X=Y, p=q, f \in \operatorname{Bir}(X)$, then we say that $f$ is $p$-fiberwise and denote by $\operatorname{Bir}_{p}(X)$ the group of all $p$ - fiberwise birational automorphisms of $X$. We write $\operatorname{Aut}_{p}(X)$ for the intersection of $\operatorname{Bir}_{p}(X)$ and $\operatorname{Aut}(X)$ in $\operatorname{Bir}(X)$, which is the group of all $p$ - fiberwise automorphisms of $X$.

- Recall that if a smooth projective variety $A$ is rigid, then any rational map from a smooth variety to $A$ is a morphism ([De, Corollary 1.44]). In particular, $\operatorname{Bir}(A)=\operatorname{Aut}(A)$. Abelian varieties and bielliptic surfaces are rigid.

We start with an auxiliary

Lemma 2.1. Assume that $U, V$ are smooth irreducible quasiprojective varieties endowed by a surjective morphism $p: U \rightarrow V$ such that the fiber $P_{v}:=p^{-1}(v)$ is projective and irreducible for every point $v \in V$. Assume that $C \subset U$ is a closed subset and that $C \cap P_{v}$ is a finite set for every point $v \in V$. Assume that $f \in \operatorname{Aut}_{p}(U \backslash C)$.

Then $f \in \operatorname{Aut}_{p}(U)$.

Remark 2.2. In loose language this Lemma asserts that every fiberwise automorphism $f \in \operatorname{Aut}(U \backslash C)$ may be extended to an automorphism of $U$ if $C$ has only " $p$-horizontal " components over $V$.

Proof. Take any smooth projective closure $\bar{V}$ of $V$ and choose such a smooth projective closure $\bar{U}$ of $U$ that the rational extension $\bar{p}: \bar{U} \rightarrow \bar{V}$ of $p$ is a morphism. Since all the fibers of $p$ are projective and irreducible, we have $\bar{p}^{-1}(V)=U, \bar{p}^{-1}(\bar{V} \backslash V)=\bar{U} \backslash U$ and $\left.\bar{p}\right|_{U}=p$ (see, for example, [MO, Section 2.6]). Let $\bar{f}: \bar{U} \rightarrow \bar{U}$ be the rational extension of $f$. Let $\left(\tilde{U}^{\prime}, \tilde{f}, \pi\right)$ be a resolution of indeterminacy of $\bar{f}$. Let $\bar{g}_{f} \in \operatorname{Bir}(\bar{V})$ be an extension of $g_{f}$.

We have a commutative diagram

$$
\begin{aligned}
& \tilde{U}^{\prime} \\
& \pi \downarrow \searrow \tilde{f} \\
& \bar{U} \quad \stackrel{\bar{f}}{-\rightarrow \bar{U}} \quad, \\
& \bar{p} \downarrow \quad \downarrow \bar{p} \\
& \bar{V} \quad \stackrel{\bar{g}_{f}}{-\rightarrow} \bar{V}
\end{aligned}
$$


Since $g_{f}$ is an automorphism of $V$, we have

$$
\tilde{U}:=(\bar{p} \circ \pi)^{-1}(V)=(\bar{p} \circ \tilde{f})^{-1}(V)=\pi^{-1}(U)
$$

and we may restrict the maps $\pi, \tilde{f}, \bar{f}, \bar{p}, \bar{g}_{f}$ to quasiprojective varieties $\tilde{U}, U$, and $V$ and obtain the following commutative diagram:

$$
\begin{array}{rrrr}
\tilde{U} & & \\
\pi \downarrow & \left.\searrow \tilde{f}\right|_{\tilde{U}} & \\
U & \left.\bar{f}\right|_{U} & \\
p \downarrow & & \\
V & \downarrow & p \\
V & \stackrel{g_{f}}{\longrightarrow} V &
\end{array}
$$

Here

- $\pi$ and $\tilde{f}:=\left.\tilde{f}\right|_{\tilde{U}}$ are morphisms;

- $f:=\left.\bar{f}\right|_{U} \in \operatorname{Aut}(U \backslash C) \cap \operatorname{Bir}(U)$;

- $\pi$ is an isomorphism of $U_{1}:=\pi^{-1}(U \backslash C)$ to $U \backslash C$.

We have to show that $f$ is defined at all points of $C$. For this, we need to check that $\tilde{f}\left(\pi^{-1}(c)\right)$ is a point for every point $c \in C$. Since $\pi$ and $\tilde{f}$ are birational morphisms, the sets $\tilde{f}^{-1}(a)$ and $\pi^{-1}(a)$ are connected for every point $a \in U$ by the Zariski Main Theorem (see [Mu1, Chapter III, §9). Take $a \in U \backslash C$. Then $\tilde{f}^{-1}(a)$ contains an isolated point $\pi^{-1}\left(f^{-1}(a)\right) \in U_{1}$, which (by the Zariski Main Theorem) is the only connected component of $\tilde{f}^{-1}(a)$. Thus $\tilde{f}^{-1}(U \backslash C)=U_{1}, \tilde{f}^{-1}(C)=\pi^{-1}(C)$, or $\tilde{f}\left(\pi^{-1}(C)\right)=C, \tilde{f}\left(U_{1}\right)=U \backslash C$. Hence for every point $c \in C$ we have

$$
\tilde{f}\left(\pi^{-1}(c)\right) \subset C \cap P_{g_{f}(p(v))}
$$

and the latter is a finite set. Since $\tilde{f}\left(\pi^{-1}(c)\right)$ has to be irreducible, it is a single point. Thus $f=\tilde{f} \circ \pi^{-1}$ is defined at every point of $U$.

Lemma 2.3. Assume that a group $G$ sits in the short exact sequence

$$
\{0\} \rightarrow G_{1} \rightarrow G \rightarrow G_{2} \rightarrow\{0\} .
$$

Suppose that one of the following two condition holds.

(1) $G_{1}$ is bounded and $G_{2}$ is strongly Jordan.

(2) $G_{1}$ is strongly Jordan and $G_{2}$ is bounded.

Then $G$ is strongly Jordan.

Proof. Suppose (1) holds. Then a lemma of Anton Klyachko [BZ2, Lemma 2.1] implies that $G$ is strongly Jordan.

Suppose (2) holds. Then both $G_{1}$ and $G_{2}$ are quasi-bounded. By Remark 1.3, $G$ is also quasi-bounded. It follows from [MZ, Lemma 2.3(1)] that $G$ is Jordan. This implies that $G$ is strongly Jordan. 
Remark 2.4. Let $X$ be a projective variety. It is actually proven in [MZ that $\operatorname{Aut}(X)$ is strongly Jordan (not just Jordan): the assertion follows readily from the combination of [MZ, Theorem 1.4] and [MZ, Lemma 2.5].

In the next Proposition we consider the group $\operatorname{Aut}_{p}(X)$ where $p: W \rightarrow A$ is a morphism from a smooth quasiprojective variety $W$ with projective fibers and $A$ is a smooth rigid projective variety.

Proposition 2.5. Suppose that $A$ is a smooth rigid projective variety of positive dimension. Let $X$ be a smooth irreducible projective variety and $p: X \rightarrow A$ morphism such that the generic fiber (and, hence, the fiber over a general point a $\in A$ ) is connected. Let $S \subsetneq A$ be a closed subset of $A$. Put $Z=p^{-1}(S)$ and $W=X \backslash Z$. Then the group $H=\operatorname{Aut}_{p}(W)$ is strongly Jordan.

Remark 2.6. Let $A_{r}$ be the set of all points $a \in A \backslash S$ such that the fiber $p^{-1}(a)$ is smooth (hence, irreducible). Then $W_{r}:=p^{-1}\left(A_{r}\right)$ is evidently $H$-invariant and $H$ is embedded in $\operatorname{Aut}\left(W_{r}\right)$. Thus while proving the Proposition we may assume that for every point $a \notin S$ the fiber $p^{-1}(a)$ is irreducible.

Proof. If $S=\emptyset$ then $W=X$ is projective and the desired result follows from results of [MZ] (and Remark 2.4). Thus we assume that $S \neq \emptyset$. Then

- We denote by $G(A):=\operatorname{Aut}(A)$ be the group of automorphisms of $A$.

- We denote by $G(S) \subset G(A)$ the subgroup of all elements $g \in G(A)$ such that $g(S)=S$;

- The identity component $G(A)_{0}$ of $G(A)$ is a connected algebraic group ([Mat, Corollary 2]) ;

- The intersection $G_{S}=G(S) \cap G(A)_{0}$ is a closed subgroup of $G(A)_{0}$, because $S$ is a closed subset of $A$;

- The identity component $G_{0}$ of $G_{S}$ is a closed subgroup in $G_{S}$, thus it is a connected algebraic group, and has finite index in $G_{S}$;

- The factor group $G(S) /\left(G_{S}\right)$ is bounded ( [MZ, Lemma 2.5]);

- Hence, the group $G(S) / G_{0}$ is bounded;

- Since $G_{0}$ acts on a non-uniruled projective variety $A$, it contains no non-trivial connected linear algebraic subgroup (otherwise, the open dense subset of $A$ would be covered by rational orbits). Thus it is isomorphic to an abelian variety by the Chevalley's Theorem $([\mathrm{C}]$ ).

By definition, for every automorphism $f \in \operatorname{Aut}_{p}(W)$ there is $g_{f} \in \operatorname{Aut}(A \backslash S)$ that may be included into the following commutative diagram :

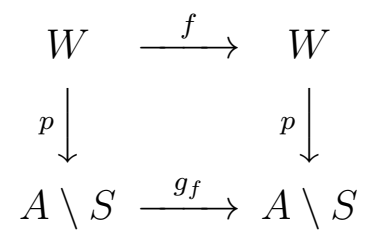


Hence, the group $H=\operatorname{Aut}_{p}(W)$ sits in the following exact sequence

$$
0 \rightarrow H_{i} \rightarrow H \rightarrow H_{a} \rightarrow 0
$$

where

$-H_{i}=\left\{f \in H \mid g_{f}=\mathrm{id}_{A}\right\}$ is a subgroup of the automorphism group of the generic fiber $\mathcal{W}_{p}$ of $p$;

$-H_{a}=\left\{g \in \operatorname{Aut}(A \backslash S) \mid g=g_{f}\right.$ for some $\left.f \in H\right\}$.

Note that we have

- $H_{a} \subset G(S)$, since $\operatorname{Bir}(A)=\operatorname{Aut}(A)$;

- Every $g \in H_{a} \subset G(S)$ moves a $G_{0}$-orbit (in $A$ ) to a $G_{0}$-orbit, since $G_{0}$ is a closed normal subgroup of $G(S)$;

- The orbit $G_{0}(z)$ of a point $z \notin S$ is a projective subset of $A$, since $G_{0}$ is an abelian variety;

- The orbit $G_{0}(z)$ of a point $z \notin S$ does not meet $S$. Hence, if $z \notin S$ then $p^{-1}\left(G_{0}(z)\right) \cap Z=p^{-1}\left(G_{0}(z) \cap S\right)=\emptyset$, i.e $p^{-1}\left(G_{0}(z)\right)$ is a closed irreducible projective subset of $W$. Indeed it is a fibration with irreducible projective fibers over a projective orbit $G_{0}(z)$ ( [Sh, Chapter 1, n.6.3, Theorem 8]).

By a theorem of M. Rosenlicht [Ros], there exist a dense open $G_{0}$-invariant subset $U \subset A$, a quasiprojective variety $V$ and a morphism $\pi: U \rightarrow V$ such that a fiber $\pi^{-1}(v)$ is precisely an orbit of $G_{0}$ for every $v \in V$. That means that $V$ is a geometric quotient of $U$ by the $G_{0}$-action. Since $S$ is $G_{0}$-invariant, we may assume that $U \subset$ $A \backslash S$. Since every $g \in H_{a} \subset G(S)$ moves a $G_{0}$-orbit (in $A$ ) to a $G_{0}$-orbit, the map $h_{g}:=\pi \circ g \circ \pi^{-1}: V \rightarrow V$ is defined at every points of $V$, hence is a morphism (see [It, Lemma 10.7 on pp. 314-315]) . Moreover, the following diagram commutes.

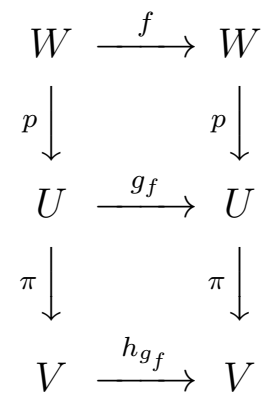

Let $\tau=\pi \circ p$. We have $f \in \operatorname{Aut}_{\tau}(W)$. Since the general fiber $T_{v}=\tau^{-1}(v), v \in V$ is a projective irreducible variety, the generic fiber $\mathcal{T}$ of $\tau$ is projective and irreducible as well ([EGA, Proposition 9.7.8.]).

Moreover, we have the following exact sequence of groups

$$
0 \rightarrow H_{T} \rightarrow H \rightarrow H_{V} \rightarrow 0
$$

where 
$-H_{T}=\left\{f \in H \mid h_{g_{f}}=\operatorname{id}_{V}\right\} ;-H_{V}=\left\{h \in \operatorname{Aut}(V) \mid h=h_{g_{f}}\right.$ for some $\left.f \in H\right\}$. (In particular case of $G_{0}=\left\{\operatorname{id}_{A}\right\}$ we have $V=A \backslash S, \pi=\operatorname{id}_{A}, h_{g_{f}}=g_{f}$, and $H_{V}=H_{a} \subset G_{S}$.) The group $H_{T} \subset \operatorname{Aut}(\mathcal{T})$ is strongly Jordan, according to [MZ, Theorem 1.4, Lemma 2.5] (and Remark 2.4). The group $H_{V}$ is isomorphic to a subgroup of $G(S) / G_{0}$, hence is bounded. Therefore, by Lemma 2.3, $H$ is strongly Jordan.

\section{Admissible triples AND RElated eXACT SEQUenCES}

Let $n$ be a positive integer and $A$ be a $n$-dimensional irreducible smooth rigid projective variety (e.g, an abelian variety or a product of curves of positive genus). We write $\mathcal{K}$ for $k(A)$.

Let us define an $A$-admissible triple as a triple $(X, \phi, Z)$ that consists of a smooth irreducible projective variety $X$, a birational isomorphism $\phi: X \rightarrow A \times \mathbb{P}^{1}$ and a closed subset $Z \subsetneq X$. We denote by $W$ the open subset

$$
W=X \backslash Z \subset X .
$$

We will freely use the following notation and properties of admissible $A$-triples.

a: Let $p_{A}: A \times \mathbb{P}^{1} \rightarrow A$ be the projection map on the first factor. Then the composition $p:=p_{A} \circ \phi: X \rightarrow A$ is a morphism, since $A$ is rigid. We say that $p$ is induced by $\phi$.

b: Since $X$ is birational to $A \times \mathbb{P}^{1}$, there is an open non-empty subset $B \subset A$ such that $\phi$ induces an isomorphism between $X_{B}=p^{-1}(B)$ and $B \times \mathbb{P}^{1}$. (This follows from the fact that indeterminancy locus of $\phi$ has codimension $\leq 2$ in $X$, thus it is mapped by $p$ into a proper closed subset of $A$.)

Moreover, $\phi$ is $p, p_{A}$-fiberwise: the following diagram commutes.

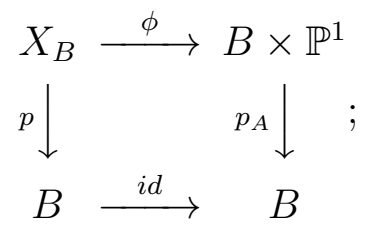

c: It follows from (6) that the general fiber $P_{x}:=p^{-1}(x)$ (i.e. fiber over a point $x$ of a certain open dense subset of $A$ ) is isomorphic to $\mathbb{P}^{1}$;

d: Let us put:

- $r(Z)$ - the number of irreducible over $\mathcal{K}$ components of $Z$ that are mapped dominantly onto $A$; we will call such components "horizontal";

- $m(Z)$ - the degree of the restriction of $p$ to $Z$, i.e the number of $\overline{\mathcal{K}}$-points in $p^{-1}(a)$ for a general point $a \in A$. 
e: The generic fiber $\mathcal{X}_{p}$ of $p$ is isomorphic to the projective line $\mathbb{P}_{\mathcal{K}}^{1}$ over $\mathcal{K}$; the generic fiber $\mathcal{W}_{p}$ of the restriction $\left.p\right|_{W} \rightarrow A$ (of $p$ to $W$ ) is isomorphic to $\mathbb{P}_{\mathcal{K}}^{1} \backslash M$, where $M$ is a finite set that is defined over $\mathcal{K}$ and consists of $m(Z)$ points that are defined over a finite algebraic extension of $\mathcal{K}$. In other words, the $\mathcal{K}$-variety $\mathcal{W}_{p}$ is isomorphic to the projective line over $\mathcal{K}$ with $m(Z)$ punctures. In particular, the group $\operatorname{Aut}_{\mathcal{K}}\left(\mathcal{W}_{p}\right)$ is finite if $m(Z)>2$. On the other hand, $r(Z)$ is the number of Galois orbits in the set of punctures. In particular,

$$
1 \leq r(Z) \leq m(Z) \text { or } 0=r(Z)=m(Z) .
$$

f: We may choose $B$ in such a way that $Z_{B}:=Z \cap X_{B}$ meets every fiber $P_{b}, b \in B$, at precisely $m(Z) \overline{\mathcal{K}}$ - points. In particular, $Z_{B}$ is a finite cover of $B$.

g: Every birational map $f \in \operatorname{Bir}(X)$ is $p$-fiberwise : we denote by $g_{f} \in \operatorname{Bir}(A)$ the corresponding automorphism $g_{f}: A \rightarrow A$ (see [BZ2]). Since $A$ is rigid, $g_{f}$ actually belongs to $\operatorname{Aut}(A)$. This implies that

$$
\operatorname{Aut}(W)=\operatorname{Aut}_{p}(W) .
$$

(Here $p$ denotes the restriction of $p: X \rightarrow A$ to $W \subset X$.)

h: Let us consider the subgroups

$$
H_{i}=\left\{f \in \operatorname{Aut}(W) \mid g_{f}=\operatorname{id}_{A}\right\} \subset \operatorname{Aut}(W)
$$

and

$$
H_{a}=\left\{g \in \operatorname{Aut}(A) \mid g=g_{f} \text { for some } f \in \operatorname{Aut}(W)\right\} \subset \operatorname{Aut}(A) .
$$

We have the following short exact sequence of groups.

$$
0 \longrightarrow H_{i} \longrightarrow \operatorname{Aut}(W) \longrightarrow H_{a} \longrightarrow 0
$$

j: Group $H_{i}$ is isomorphic to a subgroup of $\operatorname{Aut}_{\mathcal{K}}\left(\mathcal{W}_{p}\right)$. Thus it is Jordan; it is finite if $m(Z)>2$.

Remark 3.1. Let $W$ be a smooth quasiprojective irreducible variety that is birational to $A \times \mathbb{P}^{1}$. Then there is an $A$-admissible triple $(X, \phi, Z)$ such that $X \backslash Z$ is biregular to $W$. Indeed, one may take as $X$ any smooth projective closure of $W$ and put $Z=X \backslash W$.

Lemma 3.2. Suppose that $A$ is an irreducible smooth projective variety that is not uniruled. (E.g., $A$ is rigid). Then $\operatorname{Bir}\left(A \times \mathbb{P}^{1}\right)$ is quasi-bounded.

Proof. Let $p_{A}: A \times \mathbb{P}^{1} \rightarrow A$ be the projection map. Its generic fiber $\mathcal{X}$ is the projective line $\mathbb{P}_{k(A)}^{1}$ over $k(A)$. Each $u \in \operatorname{Bir}\left(A \times \mathbb{P}^{1}\right)$ is a $p_{A}$-fiberwise, see [BZ2, Lemma 3.4 and Cor. 3.6]. By [BZ2, Cor. 3.6], $\operatorname{Bir}\left(A \times \mathbb{P}^{1}\right)$ sits in an exact sequence

$$
\{0\} \rightarrow \operatorname{Bir}_{k(A)}(\mathcal{X}) \rightarrow \operatorname{Bir}\left(A \times \mathbb{P}^{1}\right) \rightarrow \operatorname{Bir}(A) .
$$

Actually, $\operatorname{Bir}\left(A \times \mathbb{P}^{1}\right) \rightarrow \operatorname{Bir}(A)$ is surjective, because one may lift any birational automorphism of $A$ to a birational automorphism of $A \times \mathbb{P}^{1}$. On the other hand, 
since $\mathcal{X}$ is the projective line, $\operatorname{Bir}_{k(A)}(\mathcal{X})$ is the projective linear group $\operatorname{PGL}(2, k(A))$. This gives us a short exact sequence

$$
\{0\} \rightarrow \operatorname{PGL}(2, k(A)) \rightarrow \operatorname{Bir}\left(A \times \mathbb{P}^{1}\right) \rightarrow \operatorname{Bir}(A) \rightarrow\{0\} .
$$

The theorem of Jordan implies that the linear group PGL $(2, k(A))$ is strongly Jordan. In particular, it is quasi-bounded. On the other hand, since $A$ is not uniruled, $\operatorname{Bir}(A)$ is also quasi-bounded ([PS1, Remark 6.9], [BZ2, Proof of Cor. 3.8 on p. 236]. It follows from (8) and Remark 1.3 that $\operatorname{Bir}\left(A \times \mathbb{P}^{1}\right)$ is also quasi-bounded.

Lemma 3.3. Assume that $m(Z)=2$ and $r(Z)=1$. Let $\operatorname{Tor}\left(H_{i}\right)$ be the set of all elements of $H_{i}$ of finite order. Then the following conditions hold.

(i) $\operatorname{Tor}\left(H_{i}\right)$ consists of, at most, 4 elements.

(ii) every element of finite order in $H_{i}$ has order 1 or 2.

(iii) Every finite subgroup of $H_{i}$ is abelian and its order divides 4 while its exponent divides 2.

Proof. Let $\left(u_{0}: u_{1}\right)$ be homogeneous coordinates in $\mathbb{P}_{\mathcal{K}}^{1}$. Since $m(Z)=2$, and $Z$ has only one irreducible component $Z_{1}$ over $\mathcal{K}$, we may assume that $Z_{1}$ is defined by equation $\left(u_{0}-\mu_{1} u_{1}\right)\left(u_{0}-\mu_{2} u_{1}\right)=0$, where $\mu_{1}, \mu_{2}$ are distinct elements of a quadratic extension $\mathcal{K}_{2}$ of $\mathcal{K}$ that are conjugate over $\mathcal{K}$.

Every automorphism $f \in \operatorname{Tor}\left(H_{i}\right)$ of $\mathcal{W}_{p}$ may be extended uniquely to a periodic automorphism $\bar{f}$ of $\mathcal{X}_{p} \cong \mathbb{P}_{\mathcal{K}}^{1}$. The 2-element subset

$$
\left\{\mu_{1}, \mu_{2}\right\} \subset \mathbb{A}^{1}\left(\mathcal{K}_{2}\right) \subset \mathbb{P}^{1}\left(\mathcal{K}_{2}\right)
$$

is $\bar{f}$-invariant for all $f \in H_{i}$. This means that either $\bar{f}$ leaves invariant both $\mu_{i}$ or permutes them.

Put $z=\frac{\left(u_{0}-\mu_{1} u_{1}\right)}{\left(u_{0}-\mu_{2} u_{1}\right)} \in \mathcal{K}_{2}(A)$. The extension $\bar{f}$ leaves the set $\{z=0\} \cup\{z=\infty\}$ invariant for all $f \in H_{i}$. Thus, $\bar{f}(z)=\lambda z$ or $\bar{f}(z)=\frac{\lambda}{z}$ for suitable nonzero $\lambda \in \mathcal{K}_{2}$. In both cases $(\bar{f})^{2}(z):=(\bar{f} \circ \bar{f})(z)=\lambda^{2} z$, This implies that $\lambda$ is a root of unity if $\bar{f}$ is periodic, i.e., if $f \in \operatorname{Tor}\left(H_{i}\right)$; in particular $\lambda \in \mathcal{K}$. Suppose that $\bar{f}\left(u_{0}: u_{1}\right)=\left(u_{0}^{\prime}: u_{1}^{\prime}\right)$. Then one may easily check that either (in the former case)

$$
\frac{\left(u_{0}^{\prime}-\mu_{1} u_{1}^{\prime}\right)}{\left(u_{0}^{\prime}-\mu_{2} u_{1}^{\prime}\right)}=\lambda \frac{\left(u_{0}-\mu_{1} u_{1}\right)}{\left(u_{0}-\mu_{2} u_{1}\right)}
$$

or (in the latter case)

$$
\frac{\left(u_{0}^{\prime}-\mu_{1} u_{1}^{\prime}\right)}{\left(u_{0}^{\prime}-\mu_{2} u_{1}^{\prime}\right)}=\lambda \frac{\left(u_{0}-\mu_{2} u_{1}\right)}{\left(u_{0}-\mu_{1} u_{1}\right)} .
$$

In order for these maps to be defined over $\mathcal{K}$ the matrices (respectively)

$$
\left(\begin{array}{cc}
\mu_{1}-\lambda \mu_{2} & \mu_{1} \mu_{2}(\lambda-1) \\
(1-\lambda) & \lambda \mu_{1}-\mu_{2}
\end{array}\right) \text { and }\left(\begin{array}{cc}
\mu_{1}-\lambda \mu_{2} & \lambda \mu_{2}^{2}-\mu_{1}^{2} \\
(1-\lambda) & \lambda \mu_{2}-\mu_{1}
\end{array}\right)
$$

should be defined (up to multiplication by a nonzero element of $\mathcal{K}_{2}$ ) over $\mathcal{K}$ as well. Since $\lambda \in \mathcal{K}$, it may happen only if $\lambda= \pm 1$. This implies that $(\bar{f})^{2}: z \mapsto \lambda^{2} z$ is the 
identity map, i.e., the order of $\bar{f}$ is either 1 or 2 . In addition, there are, at most, four elements in $\operatorname{Tor}\left(H_{i}\right)$. Namely, (written in $z$ - coordanate)

$$
\bar{f}(z)=z, \bar{f}(z)=-z, \bar{f}(z)=\frac{1}{z}, \bar{f}(z)=-\frac{1}{z} .
$$

One may see Lemma 3.3 in a more general way. Let $\mathcal{K}$ be a field of characteristic zero that contains all roots of unity. Let $n \geq 2$ be an integer.

The following assertion is an easy application of Kummer theory [La, Chapter VI, Section 8].

Theorem 3.4. Let $u$ be a matrix in $\operatorname{GL}(n, \mathcal{K})$, whose image $\bar{u}$ in $\operatorname{PGL}(n, \mathcal{K})$ has finite order. Suppose that $u$ has an eigenvalue that does not belong to $\mathcal{K}$. Then there is a positive integer $d$ such that $d \mid n$ and all eigenvalues of $u^{d}$ lie in $\mathcal{K}$. In addition, if $n$ is a prime then $\bar{u}$ has order $n$.

Proof. We know that there are a positive integer $m$ and a nonzero element $a \in \mathcal{K}$ such that $u^{m}=a \cdot \mathbf{1}_{n}$ where $\mathbf{1}_{n}$ is the identity square matrix of size $n$. Clearly, the order of $\bar{u}$ is strictly greater than 1 and divides $n$.

Let $\alpha$ be an eigenvalue of $u$ that does not belong to $\mathcal{K}$. Then $\alpha^{m}=a$. Let us consider the finite algebraic field extension $\mathcal{K}^{\prime}=\mathcal{K}(\alpha)$ of $\mathcal{K}$ and denote by $d$ its degree $\left[\mathcal{K}^{\prime}: \mathcal{K}\right]$. Clearly, $d>1$. The Kummer theory tells us that $\mathcal{K}^{\prime} / \mathcal{K}$ is a a cyclic extension and $d \mid m$. In other words, $\mathcal{K}^{\prime} / \mathcal{K}$ is Galois and its Galois group $G$ is cyclic of order $d$. If $\beta$ is another eigenvalue of $u$ then

$$
\beta^{m}=a=\alpha^{m}
$$

and therefore the ratio $\beta / \alpha$ is an $m$ th root of unity and therefore lies in $\mathcal{K}$. This implies that

$$
\mathcal{K}(\beta)=\mathcal{K}(\alpha)=\mathcal{K}^{\prime}
$$

in particular, none of eigenvalues of $u$ lies in $\mathcal{K}$.

Recall that the cardinality of $G$ coincides with $d$. Since $\beta$ generates $\mathcal{K}^{\prime}$ over $\mathcal{K}$, the set $\{\sigma(\beta) \mid \sigma \in G\}$ consists of $d$ distinct elements, each of which is an eigenvalue of $u$ and has the same multiplicity. Since the spectrum of $u$ is a disjoint union of $G$-orbits, $d$ divides $n$.

Take an element $\tau$ of (abelian group) $G$. Then $\tau(\beta)=\zeta \beta$ where $\zeta$ is a root of unity that lies in $\mathcal{K}$. The norms of conjugate $\beta$ and $\tau(\beta)$ (with repect to $\mathcal{K}^{\prime} / \mathcal{K}$ ) do coincide. This means that

$$
\prod_{\sigma \in G} \sigma(\beta)=\prod_{\sigma \in G} \sigma(\tau \beta)=\zeta^{d} \prod_{\sigma \in G} \sigma(\beta) .
$$

It follows that

$$
\zeta^{d}=1, \tau\left(\beta^{d}\right)=(\tau \beta)^{d}=\beta^{d}
$$

for all $\tau \in G$. This implies that $\beta^{d} \in \mathcal{K}$ for all eigenvalues $\beta$ of $u$ and therefore all eigenvalues of $u^{d}$ lie in $\mathcal{K}$. 
Now assume that $n$ is a prime. Then $d=n$ and counting arguments imply that the spectrum of $u$ consists of exactly one $G$-orbit say, $G \beta$. Then all the eigenvalues of $u^{n}=u^{d}$ coincide with

$$
\beta^{n}=\beta^{d} \in \mathcal{K} .
$$

This implies that $u^{n}$ is a scalar and therefore the order of $\bar{u}$ divides $n$. One has only to recall that this order is greater than 1 and $n$ is a prime. 0 .

The next lemmas show that the case $m(Z) \leq 2$ may be reduced to the case $m(Z)=$

To this end we find an open $H_{a}$-invariant subset $\tilde{B} \subset A$ of $A$ such that $\tilde{W}=p^{-1}(\tilde{B})$ is a complement of exactly two (respectively 1) "horizontal" components . Namely, we build a rank two vector bundle $E$ over $\tilde{B}$ such that $\tilde{W}$ appears to be isomorphic to the complement $Y \backslash D$ of two ( respectively, one) disjoint sections in $Y:=P(E)$.

More precisely, we are going to build the following chain of maps and inclusions of smooth irreducible quasiprojective varieties

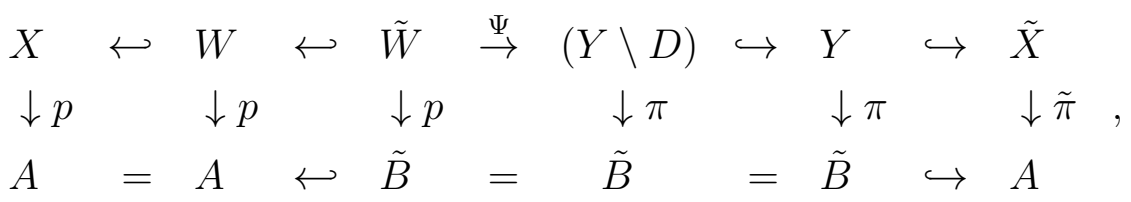

such that:

- $\tilde{B}$ is an open dense subset of $A$ invariant under the $H_{a}$-action;

- $\tilde{W}=p^{-1}(\tilde{B}) \subset W$ is invariant under the action of $\operatorname{Aut}(W)$;

- $\Psi$ is an isomorphism;

- every fiber of $\pi: Y \rightarrow \tilde{B}$ is projective;

- $D$ is a closed subset of $Y$ that meets every fiber of $\pi$ at no more than two points;

- $\tilde{X}$ is projective

- $\tilde{\pi}(\tilde{X} \backslash Y)=A \backslash \tilde{B}$.

According to Lemma 2.1, $\operatorname{Aut}(Y \backslash D) \subset \operatorname{Aut}(Y)$. Thus, instead of $\operatorname{Aut}(W)$ we may study $\operatorname{Aut}(Y)$ where $Y$ is fibered over $\tilde{B} \subset A$ with projective fibers (hence $m(\tilde{X} \backslash Y)=0)$.

The building of this construction is done in the following Lemmas.

Lemma 3.5. If $(X, \phi, Z)$ is an $A$-admissible triple, $W:=X \backslash Z$ and $m(Z)=1$ then there exists an $A$-admissible triple $(\tilde{X}, \tilde{\phi}, \tilde{Z})$ with $m(\tilde{Z})=0$ and a group embedding $\operatorname{Aut}(W) \hookrightarrow \operatorname{Aut}(\tilde{W})$, where $\tilde{W}:=\tilde{X} \backslash \tilde{Z}$.

Proof. Let $\left(w_{0}: w_{1}\right)$ be homogeneous coordinates in $\mathbb{P}^{1}$. We may choose them in such a way that $\phi\left(Z_{B}\right)=B \times\{(0: 1)\}$.

Let

- $W_{B}=W \cap X_{B}=X_{B} \backslash Z_{B}$;

- $B_{g}=g(B)$ for an automorphism $g \in H_{a}$; 
- $W_{g}=W \cap p^{-1}\left(B_{g}\right)$;

- $\tilde{B}=\cup B_{g}, g \in H_{a}$;

- $\tilde{W}=p^{-1}(\tilde{B})=\cup W_{g}, g \in H_{a}$.

We have $\phi\left(W_{B}\right)=B \times\left(\mathbb{P}^{1} \backslash\left\{w_{0}=0\right\}\right)$. Thus, the rational function $t=w_{1} / w_{0}$ is defined on $\phi\left(W_{B}\right)$ and the rational function $\tau=\phi^{*}(t)$ is defined on $W_{B}$. It establishes an isomorphism of the fiber $p^{-1}(b) \cap W$ with $\mathbb{A}_{t}^{1}$ if $b$ is a point of $B$.

For every $g \in H_{a}$ there exists $f_{g} \in \operatorname{Aut}(W)$ such that $g=g_{f_{g}}$ and $f_{g}\left(W_{B}\right)=W_{g}$. We define $\tau_{g}=\tau \circ f_{g}^{-1}$, which is a regular function on $W_{g}$. (Note that apriori the choice of $f_{g}$ is not unique. A different choice of $f_{g}$ will change $\tau_{g}$ by a $p$-fiberwise automorphism of $W_{B}$, which becomes a nondegenerate affine transformation of the generic fiber $\mathcal{W}_{p} \sim \mathbb{A}_{\mathcal{K}}^{1}$.)

We introduce the isomorphisms $\psi_{g}: W_{g} \rightarrow B_{g} \times \mathbb{A}^{1}$ by $\psi_{g}(w)=\left(p(w), \tau_{g}(w)\right)$. Actually, $\psi_{g}$ are compositions of the chain of automorphisms

$$
W_{g} \stackrel{f_{g}^{-1}}{\rightarrow} W_{B} \stackrel{\phi}{\rightarrow} \quad B \times \mathbb{A}_{t}^{1} \stackrel{\left(g^{-1}, i d\right)}{\rightarrow} B_{g} \times \mathbb{A}_{t}^{1} .
$$

Note that in this chain $f_{g}^{-1}$ is $p$-fiberwise, $\phi$ is $p, p_{A}$-fiberwise, and $\left(g^{-1}\right.$, id $)$ is $p_{A}$-fiberwise, thus $\psi_{g}$ is $p, p_{A}$-fiberwise. It may be included into the following commutative diagram

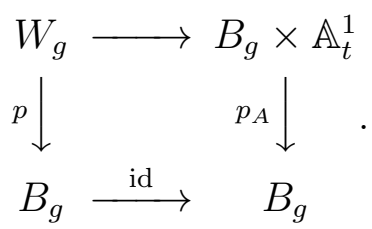

If $g, h \in H_{a}$ and $w \in W_{g} \cap W_{h}$ then:

- $b=p(w) \in\left(B_{g} \cap B_{h}\right)$;

- functions $\tau_{g}$ and $\tau_{h}$ provide an isomorphism of the fiber $P_{b}=p^{-1}(b) \cap W$ with $\mathbb{A}_{t}^{1}$ hence

$$
\tau_{g}=\tau \circ f_{g}^{-1}=\tau \circ f_{h}^{-1} \circ f_{h} \circ f_{g}^{-1}=\tau_{h} \circ f_{h} \circ f_{g}^{-1}=\tau_{h} \alpha+\beta,
$$

where $\alpha:=\alpha_{g h}(b), \beta:=\beta_{g h}(b)$ are regular in $B_{g} \cap B_{h}$, constant along $P_{b}$, and $\alpha_{g h}$ does not vanish in $\left(B_{g} \cap B_{h}\right)$;

- $\Psi_{g h}=\psi_{g}(w) \circ \psi_{h}^{-1}$ is a $p_{A}$-fiberwise automorphism of $\left(B_{g} \cap B_{h}\right) \times \mathbb{A}^{1}$ defined by $\Psi_{g h}\left(b, \tau_{h}\right)=\left(b, \tau_{g}\right)=\left(b, \alpha_{g h}(b) \tau_{h}+\beta_{g h}(b)\right)$;

It follows that $\tilde{W}$ is the total body of an $\mathbb{A}^{1}$-bundle on $\tilde{B}$ : the latter is defined by transition functions $\Psi_{g h}$.

We define a rank two vector bundle by the following data.

- the covering of $\tilde{B}$ by the open subsets $B_{g}, g \in H_{a}$;

- natural projection $\pi_{E}: B_{g} \times \mathbb{A}_{\left(u_{0}, u_{1}\right)}^{2} \rightarrow B_{g}$; 
- transition matrices on $B_{g} \cap B_{h}$

$$
M_{g h}=\left(\begin{array}{cc}
1 & 0 \\
\beta_{g h} & \alpha_{g h}
\end{array}\right) .
$$

The maps

$$
\left\{\bar{\psi}_{g}(w): W_{g} \rightarrow \mathbb{P}(E), \bar{\psi}_{g}(w)=\left(p(w),\left(1: \tau_{g}(w)\right)\right.\right.
$$

glue together to an isomorphism

$$
\Psi: \tilde{W} \cong \mathbb{P}(E) \backslash\left\{u_{0}=0\right\} .
$$

We denote by $D$ the divisor (image of the section) $\left\{u_{0}=0\right\}$ in $\mathbb{P}(E)$ and by $\pi$ the induced by $\pi_{E}$ the projection map $P(E) \rightarrow \tilde{B}$.

We have $\operatorname{Aut}(W) \subset \operatorname{Aut}(\tilde{W})$, since the (sub)set $\tilde{B} \subset A$ is invariant under the action of $H_{a}$. On the other hand, according to Lemma 2.1, $\operatorname{Aut}(\tilde{W}) \subset \operatorname{Aut}(Y)$ where $Y:=\mathbb{P}(E)$. Take any smooth projective closure $\tilde{X}$ of $Y$ and extend $\pi$ to the rational map $\tilde{\pi}: \tilde{X} \rightarrow A$. Since $A$ contains no rational curves, $\tilde{\pi}$ is a morphism, which is obviously projective. Let $\tilde{D}$ be the closure of $D$ in $\tilde{X}$. Note that $\tilde{D} \cap Y=D$, $\tilde{\pi}^{-1}(\tilde{B})=Y$, and $\tilde{p}^{-1}(A \backslash \tilde{B})=\tilde{X} \backslash Y$, in light of the "maximality" property of projective (and therefore proper) morphism $\pi$ [MO, Section 2.6, pp. 95-96]).

Let $\tilde{Z}=\tilde{X} \backslash Y$. Let $\tilde{\phi}: \tilde{X} \rightarrow A \times \mathbb{P}^{1}$ be the rational extension of $\phi \circ \Psi^{-1}: Y \rightarrow$ $A \times \mathbb{P}^{1}$. Then $m(\tilde{Z})=0$, and the $A$-admissible triple $(\tilde{X}, \tilde{\phi}, \tilde{Z})$ is the one we were looking for.

Remark 3.6. This Lemma may be derived from general results in [KW] and [Su1], Su2 but we prefer an explicit construction which we use in the next Lemma.

Lemma 3.7. Assume that a triple $(X, \phi, Z)$ is $A$-admissible, $m(Z)=2$ and $r(Z)=$ 2. Then there exists an A-admissible triple $(\tilde{X}, \tilde{\phi}, \tilde{Z})$ with $m(\tilde{Z})=0$ and a group embedding $\operatorname{Aut}(W) \hookrightarrow \operatorname{Aut}(\tilde{W})$, where $\tilde{W}:=\tilde{X} \backslash \tilde{Z}$.

Proof. Since $Z_{B}$ contains two disjoint irreducible over $\mathcal{K}$ horizontal components we may choose homogeneous coordinates $\left(w_{0}: w_{1}\right)$ in $\mathbb{P}^{1}$ in such a way that $\phi\left(Z_{B}\right)=$ $B \times\left\{w_{0} w_{1}=0\right\}$. Thus this is the special case of Lemma 3.5 when (in the notation of Lemma 3.5) $\tau_{g}=0$ whenever $t=0$ for all $g \in H_{a}$. Thus this lemma follows from Lemma 3.5. Note that in this case $\beta_{g h} \equiv 0$ and instead of the $\mathbb{A}^{1}$-bundle we have a line bundle.

It follows that the case $m(Z) \leq 2$ may be reduced to the case $m(Z)=0$.

Lemma 3.8. If a triple $(X, \phi, Z)$ is A-admissible, $W:=X \backslash Z$ and $m(Z)=0$ then there exists an $A$-admissible triple $(\tilde{X}, \tilde{\phi}, \tilde{Z})$

such that:

1) There is a group embedding $\operatorname{Aut}(W) \hookrightarrow \operatorname{Aut}(\tilde{W})$ where $\tilde{W}=\tilde{X} \backslash \tilde{Z}$;

2) If $\tilde{p}$ is the projection map from $\tilde{X}$ onto $A$ induced by $\tilde{\phi}$, then $\tilde{Z}=\tilde{p}^{-1}(S)$ for a certain closed subset $S$ of $A$; in addition, for every point $b \in B:=A \backslash S$ the fiber $P_{b}=\tilde{p}^{-1}(b)$ is an irreducible reduced curve isomorphic to $\mathbb{P}^{1}$. 
Remark 3.9. In loose words it means that we can add to $Z$ all the singular fibers of $p$ without reducing an automorphism group.

Proof. Since $m(Z)=0$, we have $Z_{A}=p(Z) \neq A$ is a closed subset of $A$. Let $a$ be a point of $Z_{A}$. Then the fiber $P_{a} \cap W=P_{a} \backslash\left(Z \cap P_{a}\right)$ either has a non-projective irreducible component or is empty. Let $S_{s}$ be the set of all points $a \in A \backslash Z_{A}$ such that $P_{a}=p^{-1}(a)$ is singular (namely, has several irreducible components or a non-reduced component). Let $S:=S_{s} \cup Z_{A} \subset A$, i.e, $B:=A \backslash S$ is the set of all points $a \in A$ such that the fiber $P_{a} \subset W$ is a reduced irreducible smooth curve isomorphic to $\mathbb{P}^{1}$. Then sets $B$ and $S$ are invariant under the action of $H_{a}$ (see (7)), thus $\tilde{W}:=p^{-1}(B)$ is invariant under the action of $\operatorname{Aut}(W)$, i.e, $\operatorname{Aut}(W) \subset \operatorname{Aut}(\tilde{W})$.

Thus, the $A$-admissible triple

$$
\tilde{X}:=X, \tilde{\phi}:=\phi, \tilde{Z}:=p^{-1}(S)
$$

enjoys the desired properties.

\section{Proof of Theorem 1.5}

In this section we prove Theorem 1.5 and Corollary 1.6 ,

Proof of Theorem 1.5. By Remark 1.7 we may assume that $W$ is smooth. When $W$ is projective, the desired result follows from [MZ]. So, we may assume that quasiprojective $W$ is not projective. By Remark 3.1 we may choose such an $A$-admissible $(X, \phi, Z)$ that $W=X \backslash Z$. We use the exact sequence (7).

It is proven in [PS1, Section 6] (see also [BZ2, Corollary 3.8]) that for an irreducible non-uniruled variety $A$ the group $\operatorname{Bir}(A)$ (and, hence, Aut $(A)$ ) is strongly Jordan.

If $m(Z)>2$, then the (sub)group $H_{i}$ in the short exact sequence (7) is finite. If $m(Z)=2$, and $r(Z)=1$, then, according to Lemma 3.3. $H_{i}$ is bounded. It follows from Lemma 2.3 that in both cases $\operatorname{Aut}(W)$ is Jordan. (See also [PS1, Lemma 2.8].)

According to Lemma 3.7, Lemma 3.5, Lemma 3.8, in all other cases one may assume that conditions of Proposition 2.5 are satisfied, hence, Aut $(W)$ is strongly Jordan.

Proof of Corollary 1.6. Assume that $W$ is a quasiprojective irreducible variety of dimension $d \leq 3$. The case $\operatorname{dim} W \leq 2$ was done in [Po1, Za2, BZ1]. Assume that $W$ is not birational to $E \times \mathbb{P}^{2}$, where $E$ is an elliptic curve.

If $\operatorname{dim}(W)=3$ and $\operatorname{Bir}(W)$ is Jordan, then its subgroup $\operatorname{Aut}(W)$ is also Jordan. If $\operatorname{Bir}(W)$ is not Jordan, then according to PS2, the variety $W$ has to be birational to $S \times \mathbb{P}^{1}$, where $S$ is a surface that enjoys one of the following three properties.

Case 1. $S$ is an abelian surface. Since $S$ contains no rational curves, it is rigid. Thus,Aut $(W)$ is Jordan by Theorem 1.5.

Case 2. $S$ is bielliptic surface. Since $S$ contains no rational curves, it is rigid. Thus, Aut $(W)$ is Jordan by Theorem 1.5.

Case 3. $S$ is a surface with Kodaira dimension $\varkappa(S)=1$ such that the Jacobian fibration of the pluricanonical fibration is locally trivial in Zariski topology. 
Consider Case 3. Further on we assume that $k=\mathbb{C}$.

We have to prove that $S$ is rigid. Since Jacobian fibration of the pluricanonical fibration is locally trivial in Zariski topology, all fibers (even the multiple ones) of the pluricanonical fibration are smooth elliptic curves ([Sh, Chapter VII, section 7, Corollary 2], [C-D, Theorem 5.3.1]).

Lemma 4.1. Assume that $A$ is a smooth irreducible surface endowed with a morphism $\pi: A \rightarrow C$ such that

- $C$ is a smooth curve of genus g;

- Every fiber $F_{c}=\pi^{-1}(c), c \in C$ is a smooth elliptic curve;

- Kodaira dimension $\varkappa(A)=1$.

- Morphism $\pi$ is a pluricanonical fibration, i.e for some $N$ and every effective divisor $D \in\left|N K_{A}\right|$ there are positive numbers $\nu_{1}, \ldots, \nu_{n}$ and fibers $F_{1}, \ldots, F_{n}$ of $\pi$ such that $D=\sum_{1}^{n} \nu_{i} F_{i}$.

Then surface A contains no rational curves.

Proof. The surface $A$ enjoys the following properties:

- Euler characteristics $e(A)=0$ ( see [Sh, Chapter IV, section 4, Theorm 6]);

- Since $K_{A}^{2}=0$, we have $\chi(A)=\chi\left(A, \mathcal{O}_{A}\right)=0$ ( see [Sh] $)$;

- If fibration $\pi$ has precisely $k$ multiple fibers $F_{1}, \ldots, F_{k}$ with multiplicities $m_{1}, \ldots, m_{k}$, respectively, then

$$
\delta(\pi):=2 g-2+\sum_{i=1}^{i=k}\left(1-\frac{1}{m_{i}}\right)>0 ;
$$

(see [BHPV, Chapter V, proposition 12.5])

- In particular, $2 g-2+k>0$.

- Since $\pi$ is a pluricanonical fibration every automorphism $\phi \in \operatorname{Aut}(A)$ is $\pi$-fiberwise.

- For every automorphism $\phi \in \operatorname{Aut}(A)$ the subset $F_{\text {sing }}=F_{1} \cup \cdots \cup F_{k}$ is invariant since multiple fibers go to multiple fibers;

Let $B \subset A$ be a rational curve. Since it cannot be contained in a fiber of $\pi$, it is mapped by $\pi$ onto $C$ with some degree $m \geq 1$. Hence $C$ is rational. Assume that $B$ intersects $F_{i}$ at points $a_{1}^{i}, \ldots, a_{n_{i}}^{i}$ that are ramification points of restriction $\tilde{\pi}$ of $\pi$ onto $B$, of orders $r_{i, 1} \ldots, r_{i, n_{i}}$, respectively. Then

- $r_{i, 1}+\cdots+r_{i, n_{i}}=m$,

- $r_{i, j} \geq m_{i} j=1, \ldots, n_{i}$;

- $n_{i} \leq \frac{m}{m_{i}}$.

Assume that $\tilde{\pi}$ has also ramification points $b_{1}, \ldots, b_{r}$ of orders $p_{1}, \ldots, p_{r}$ respectively, (including nodes of $B$ ) outside $F_{\text {sing }}$. 
By the Hurwuitz formula we have

$$
\begin{gathered}
2=2 m-\sum_{i=1}^{i=k} \sum_{j=1}^{j=n_{i}}\left(r_{i, j}-1\right)-\sum_{l=1}^{l=r}\left(p_{l}-1\right)= \\
2 m-m k+\sum n_{i}-\nu,
\end{gathered}
$$

where $\nu:=\sum_{l=1}^{l=r}\left(p_{l}-1\right)$ is a non-negative number.

Thus, dividing by $m$ we get

$$
k-2=\frac{1}{m}\left(-2+\sum n_{i}-\nu\right) \leq \sum \frac{1}{m_{i}}
$$

and

$$
\delta(\pi):=-2+\sum_{i=1}^{i=k}\left(1-\frac{1}{m_{i}}\right)=-2+k-\sum \frac{1}{m_{i}} \leq 0
$$

which contradicts to (12)

Thus, in Case 3 surface $S$ is rigid as well, and $\operatorname{Aut}(W)$ is Jordan by Theorem 1.5.

Remark 4.2. In the course of the proof of Theorem 1.5 and Corollary 1.6 it suffices to consider the case when the ground field is the field $\mathbb{C}$ of complex numbers. Indeed, suppose that we know that the Theorems hold true when the ground field is $\mathbb{C}$. Let $k$ be any algebraically closed field of characteristic 0 and an algebraic variety $W$ over $k$ satisfies the conditions either of Theorem 1.5 or Corollary 1.6. Let us assume that $\operatorname{Aut}(W)$ is not Jordan. We need to arrive to a contradiction.

The variety $W$ is defined over a subfield $k_{0}$ (of $k$ ) such that $k_{0}$ is finitely generated over the field $\mathbb{Q}$ of rational numbers, i.e., there is a quasiprojective variety $W_{0}$ over $k_{0}$ such that $W=W_{0} \times_{k_{0}} k$. (Clearly, $k_{0}$ is a countable field.) Replacing if necessary $k_{0}$ by its finitely generated extension, we may assume that there is a surface $A_{0}$ over $k_{0}$ and a $k_{0}$-birational map between $W$ and $A_{0} \times \mathbb{P}^{1}$. Moreover, we may choose $k_{0}$ in such a way that

- if $A$ is bielliptic, the same is valid for $A_{0}$ (the bielliptic structure would be defined over $k_{0}$ );

- if $\varkappa(A)=1$, the same is valid for $A_{0}$ (the pluricanonical fibration would be defined over $\left.k_{0}\right)$;

- if a pluricanonical fibration of $A$ has smooth irreducible elliptic fibers, the same is valid for $A_{0}$ (smoothness is preserved under base change [Li, Proposition3.38, Chapter 4])

- if $A$ contains no rational curves the same is valid for $A_{0}$. Indeed, if $A_{0}$ contained a rational curve, then for some integer $d$ one of the irreducible quasiprojective components of the variety $\operatorname{RatCurves}_{d}^{n}(A)$ (see [Ko, Definition 2.11]) would 
have a point over $\mathbb{C}$. But then it would have a point over $k$ as well, since $k$ is algebraically closed.

The non-Jordanness of $\operatorname{Aut}(W)$ means that there exists an infinite sequence of finite subgroups $\left\{G_{i} \subset \operatorname{Aut}(W)\right\}_{i=1}^{\infty}$, whose Jordan indices $J_{G_{i}}$ tend to infinity. For each positive $i$ there is a subfield $k_{i}$ of $k$ that contains $k_{0}$ and is finitely generated over $k_{0}$, and such that all automorphisms from $G_{i}$ are defined over $k_{i}$. Clearly, all $k_{i}$ are countable fields. The compositum of all $k_{i}$ 's (in $k$ ) is countably generated over $k_{0}$ and therefore is also a countable field. Let us consider the algebraic closure $k_{\infty}$ of this compositum in $k$. Clearly, $k_{\infty}$ is an algebraically closed countable subfield of $k$ that contains all $k_{i}$. Let us consider the quasiprojective variety $W_{\infty}=W_{0} \times_{k_{0}} k_{\infty}$. Clearly, there exist group embeddings $G_{i} \hookrightarrow \operatorname{Aut}_{k_{\infty}}\left(W_{\infty}\right)$ for all positive $i$. This implies that $\operatorname{Aut}_{k_{\infty}}\left(W_{\infty}\right)$ is not Jordan.

Since $k_{\infty}$ is countable, there is a field embedding $k_{\infty} \hookrightarrow \mathbb{C}$. Let us consider the complex quasiprojective variety $W_{\mathbb{C}}=W_{\infty} \times_{k_{\infty}} \mathbb{C}$, which is birational to $A_{\mathbb{C}} \times \mathbb{P}^{1}$ where $A_{\mathbb{C}}=A \times_{k_{0}} \mathbb{C}$ is a complex variety meeting conditions of Theorem 1.5. In particular, $\operatorname{Aut}\left(W_{\mathbb{C}}\right)$ is Jordan. On the other hand, there is a group embedding $\operatorname{Aut}\left(W_{\infty}\right) \hookrightarrow \operatorname{Aut}\left(W_{\mathbb{C}}\right)$. This implies that $\operatorname{Aut}\left(W_{\mathbb{C}}\right)$ is not Jordan as well, which gives us the desired contradiction.

\section{REFERENCES}

[BHPV ] W. Barth, K. Hulek, C. Peters, A. van de Ven, Compact Complex Surfaces. SpringerVerlag, Berlin, 2004.

[BZ1] T. Bandman, Yu. G. Zarhin, Jordan groups and algebraic surfaces. Transformation Groups 20 (2015), 327-334.

[BZ2] T.Bandman, Yu. G. Zarhin, Jordan groups, conic bundles and abelian varieties . Algebraic Geometry 4:2 (2017), 229-246.

[Bir] C. Birkar, Singularities of linear systems and boundedness of Fano varieties. 2016, arXiv:1609.05543,

[BM] E. Bombieri, D. Mumford, Enriques' Classification of surfaces in Char. p, II. In: Complex Analysis and Algebraic Geometry (W.L.Baily Jr., T. Shioda, eds.), Cambridge Univ. Press, 1977, pp. 23-43.

[C] B. Conrad, A Modern Proof of the Chevalley's Theorem on Algebraic Groups. J. Ramunajam Math. Soc., 17 (2002), no. 1, 1-18.

[C-D] F. Cossec, I. Dolgachev, Enriques surfaces I. Birkhauser, Berlin, 1989.

[CR] C.W. Curtis, I. Reiner, Representation Theory of Finite Groups and Associative Algebras. Wiley, New York, 1962.

[De] O. Debarre, Higher-Dimensional Algebraic Geometry, Springer-Verlag, New York, 2001.

[Gr1] A. Grothendieck, Technique de construction et théormes d'existence en géométrie algébrique IV: les schémas de Hilbert, Séminaire N. Bourbaki, 1960-1961, exp. 221, 249-276.

[EGA] A. Grothendieck, Éléments de géométrie algébrique (rédigés avec la collaboration de $J$. Dieudonné): IV, Étude locale des schémas et des morphismes de schémas, Troisiéme partie. Publ. Math. IHES 28 (1966).

[It] Sh. Iitaka, Algebraic Geometry, GTM 76. Springer-Verlag, Berlin Heidelberg New York, 1982

$[\mathrm{KW}]$ T. Kambayashi, D. Wright, Flat families of affine lines are affine-line bundles. Illinois J. Math. 29 (1985), no. 4, 672-681. 
[Ko] J. Kollar, Rational curves on algebraic varieties. Ergebnisse der Math. 3 Folge 32, SpringerVerlag, Berlin Heidelberg New York, 1996.

[La] S. Lang, Algebra, 2nd edition. Addison-Wesley, Reading, MA, 1993.

[Li] Q. Liu, Algebraic Geometry ang Arithmetic Curves. Oxford graduate texts in Mathematics, 6, New York, 2002.

[Mat] T. Matsusaka, Polarized Varieries, Fields of Moduli and generalized Kummer Varieties of Polarized varieties. American J. Math., 80, no. 1, 45-82.

[MO] D. Mumford, T. Oda, Algebraic Geometry II. Texts and Reading in Mathematics, 73, Hindustan Book Agency, Mumbai, 2015.

[MZ] Sh. Meng, D.-Q. Zhang, Jordan property for non-linear algebraic groups and projective varieties. American J. Math., to appear; arXiv: 1507.02230 [math.AG] .

[Mu1] D. Mumford, The Red Book of Varieties and Schemes. Lecture Notes in Math. vol. 1358, Springer, 1999.

[Mu2] D. Mumford, Abelian Varieties, 3rd edition. Hindustan Book Agency, India, Mumbai, 2008.

[MuTu] I. Mundet i Riera, A.Turull, Boosting An Analogue of Jordan's Theorem For Finite Groups. Adv. Math. 272 (2015), 820-836.

[Po1] V.L. Popov, On the Makar-Limanov, Derksen invariants, and finite automorphism groups of algebraic varieties, pp. 289-311. In: Affine algebraic geometry: the Russell Festschrift. CRM Proceedings and Lecture Notes 54, Amer. Math. Soc., 2011.

[Po2] V.L. Popov, Jordan groups and automorphism groups of algebraic varieties. In: Automorphisms in Birational and Affine Geometry, Springer Proceedings in Mathematics and Statistics 79 (2014), 185-213.

[PS0] Yu. Prokhorov and C. Shramov, Jordan property for Cremona groups. Amer. J. Math. 138 (2016), no. 2, 403-418.

[PS1] Yu. Prokhorov and C. Shramov, Jordan Property for groups of birational Selfmaps. Compositio Math. 150 (2014), 2054-2072.

[PS2] Yu. Prokhorov, C. Shramov, Finite groups of birational selfmaps of threefolds. arXiv:1611.00789.

[Ros] M. Rosenlicht, A Remark on Quotient Spaces. An. Acad. Brasil Ci, 35 (1963), 487-489.

[Sa] F. Sakai, Kodaira Dimension of Complements of Divisors, pp.239-259. In: Complex Analysis and Algebraic Geometry (W.L.Baily Jr., T. Shioda, eds.), Cambridge University Press, 1977.

[Se] F. Serrano, Divisors of Bielliptic surfaces and Embedding in $\mathbb{P}^{4}$. Math. Z. 203 (1990), 527533.

[Ser] J.-P. Serre, Finite groups: an introduction. International Press, Somerville, MA, 2016.

[Sh] I.R. Shafarevich et al., Algebraic Surfaces. Proc. Steklov Inst. Math. 75, Moscow, 1965; American Mathematical Society, Providence, RI, 1967.

[Su1] H. Sumihiro, Equivariant completion. J. Math. Kyoto Univ. 14 (1974), 1-28.

[Su2] H. Sumihiro, Equivariant completion. II. J. Math. Kyoto Univ. 15 (1975), no. 3, 573-605.

[Za1] Yu.G. Zarhin, Theta groups and products of abelian and rational varieties. Proc. Edinburgh Math. Soc. 57:1 (2014), 299-304.

[Za2] Yu.G. Zarhin, Jordan groups and elliptic ruled surfaces. Transformation Groups 20 (2015), no. $2,557-572$.

Department of Mathematics, Bar-Ilan University, 5290002, Ramat Gan, ISRAEL

E-mail address: bandman@macs.biu.ac.il

Department of Mathematics, Pennsylvania State University, University Park, PA 16802, USA

E-mail address: zarhin@math.psu.edu 